\title{
Is an intestinal permeability test a valid marker for slight dietary transgressions in adolescents with coeliac disease?
}

\author{
P Fernandez-Calle, R Codoceo, I Polanco, J Gomez-Cerezo, M Orsi, J M Tenias
}

\begin{abstract}
Adolescents with coeliac disease often fail to adhere to a strict gluten free diet. The effectiveness of intestinal permeability to sugars as a marker of slight dietary transgressions by such adolescents was assessed. Severe dietary transgressions were excluded from the study. Subjects were divided into two groups according to whether they commited slight dietary transgressions or adhered to a strict gluten free diet. A reference group of preadolescents with coeliac disease was also included in the study. Intestinal permeability and antigliadin antibody tests were performed on all patients. The diagnostic marker of intestinal permeability was excellent in the reference group. Neither the intestinal permeability test nor antigliadin antibody tests, however, succeeded in discriminating between the two groups of adolescents considered in this study. In conclusion the intestinal permeability test is not a valid marker for slight dietary transgression in such patients.

(Gut 1993; 34: 774-777)
\end{abstract}

The specific treatment of coeliac disease is strict adherence to a gluten free diet. ${ }^{1}$ Such diets are not always properly followed by patients. Good dietary control is crucial for remission of the disease as well as for prevention of the development of malignant pathology of the small intestine in adults. ${ }^{23}$

Because of their psychosociological make up, adolescents present the highest dietary transgression rate of all age groups. ${ }^{45}$ Dietary transgressions may be too minor to produce clinical or biochemical effects. Small transgressions do not, however, prevent future complications, ${ }^{25-7}$ hence it would be useful to find a sensitive marker capable of detecting slight dietary transgressions.

Antigliadin, antiendomysial, and human jejunum antibodies have been proposed as such markers, ${ }^{89}$ but they are of little value in adolescents with coeliac disease, in whom, through an as yet unidentified mechanism, sensitivity is low. ${ }^{10}{ }^{11}$ Also measurements of antiendomysial or human jejunum antibodies is not feasible at all laboratories.

Intestinal permeability has also been proposed as a reliable test for the detection of villous atrophy in coeliac disease. ${ }^{12-17}$

Certain workers have suggested this method as a screening test in gluten challenge or in the detection of dietary non-compliance. ${ }^{1819}$ Our study attempts to assess intestinal permeability as a marker of slight dietary transgressions in a population of adolescents with coeliac disease.

\section{Patients and methods}

\section{STUDY SUBJECTS}

Subjects were selected from among adolescents with coeliac disease who attended the gastroenterology output service of the hospital.

Inclusion criteria were: Confirmed diagnosis of coeliac disease according to the European Society of Paediatric Gastroenterology and Nutrition (ESPGAN) ${ }^{20}$ and voluntary consent to participate in the study.

Exclusion criteria were severe intestinal involvement suggesting major dietary transgressions assessed by clinical or biochemical alterations (total faecal fat $>6 \mathrm{~g} / 24 \mathrm{~h}$ as described by Van de Kamer, ${ }^{21}$ faecal nitrogen $>1.5$ $\mathrm{g} / 24 \mathrm{~h}$ as described by Lowry et $a l,{ }^{22}$ and faecal chymotrypsin activity $<18 \mathrm{IU} / \mathrm{g}$ of dry stool (test combination Boehringer-Mannheim, Mannheim, Germany).

The selection requirements were fulfilled by 29 patients (15 males and 14 females) ranging from 14 to 20 years of age.

Selected patients were divided into subgroups on the basis of a comprehensive dietary questionnaire: subgroup I, nine patients (four males and five females) who admitted to occasional transgressions in the preceding six months; subgroup II; 20 patients ( 11 males and nine females) who asserted that they had strictly adhered to a gluten free diet.

\section{REFERENCE GROUP}

Previously we analysed a group of 30 patients who were undergoing diagnostic testing for coeliac disease. The aim of this part of the study was to establish the exact level of confidence in the ability of the intestinal permeability test to identify gluten induced villous atrophy. These patients were also divided into two subgroups: subgroup A, 19 patients from three to 10 years of age (12 males and seven females). All were on a gluten free diet and their intestinal biopsy results were normal (the second biopsy in their diagnostic series). On conclusion of the study, diagnoses of coeliac disease were confirmed for all these patients; Subgroup B, nine patients (six males and three females) aged between three and 13. All were in the gluten challenge period and their third biopsy showed severe villous atrophy. 
METHODS

The intestinal permeability test was performed within a maximum of two days after the dietary questionnaire was given. In the reference group, the test was performed three days after intestinal biopsy.

After an overnight fast (that continued until the end of the test) and emptying of the bladder, a mixture of $5 \mathrm{~g}$ lactulose and $1 \mathrm{~g} \mathrm{~L}$-rhamnose diluted in $60 \mathrm{ml}$ water was given. Urine was collected for a five hour period and patients were allowed to drink (water only) after three hours.

The volume of urine excreted during the five hour period was recorded and an aliquot of $25 \mathrm{ml}$ for each subject was stored in a plastic tube with merthiolate $(10 \% \mathrm{w} / \mathrm{v})$ as preservative at $-30^{\circ} \mathrm{C}$ until analysis.

Sugar concentration in the urine was determined by high performance liquid chromatography (HPLC) with the method described by Delahunty and Hollander ${ }^{23}$ modified slightly by replacing the last two centrifugation steps with a single filtration procedure. The HPLC equipment (Waters, Division of Millipore, Waterford, MA, USA) included a model 6000A pump, an automatic model WISP 712 injector, and a model 410 refraction index detector. The column was a Sugar-Pack I calcium form resin column and the mobile phase was grade I water/0.001 $\mathrm{mM}$ disodium calcium ethylenediaminetetra-acetic acid at a flow rate of $0.5 \mathrm{ml} / \mathrm{min}$. Calibration was peformed with an internal standard of cellobiose. Peak areas were integrated by computer (NEC Power Mate I, Nec Co, USA). Accuracy of the method was: intrabatch: $4 \cdot 26 \%$ for lactulose and $4 \cdot 82 \%$ for L-rhamnose; between day: $6 \cdot 46 \%$ and $5 \cdot 7 \%$. Recovery of both sugars was $84 \%$. Sensitivity was $1064 \mathrm{mvol} / \mathrm{mmol} / 1$ for lactulose and $356 \mathrm{mvol} / \mathrm{mmol} / \mathrm{l}$ for L-rhamnose. The slope ranged from 0.49 to $1.46 \mathrm{mmol} / \mathrm{l}(\mathrm{y}=-0.027+$ $\left.0.72 \mathrm{x}, \mathrm{r}^{2}=0.999\right)$ for lactulose and $1.94 \mu \mathrm{mol} / \mathrm{l}$ to $3.04 \mathrm{mmol} / \mathrm{l}\left(\mathrm{y}=-0.88+0.81 \mathrm{x}, \mathrm{r}^{2}=0.999\right)$ for $\mathrm{L}$ rhamnose. The only interference was produced by galactose and D-xylose, which coeluted with L-rhamnose.

Simultaneous IgA and IgG analyses of antigliadin antibodies were performed by enzyme linked immunosorbent assay microtitre (Pharmacia Diagnostic, Sweden) with $25 \mathrm{U} / \mathrm{ml}$ as the upper limit to the reference range.

A breath hydrogen test was given to all patients during the intestinal permeability test at basal metabolism, at 15 minutes, and then every 30 minutes for 210 minutes, and the results were quantified on an exhaled hydrogen monitor (GMI Medical LTd, Renfrew, England). The aim of this test was to assess whether altered orocaecal transit times or colonisation of the upper intestine might interfere with the reliability of

TABLE I Descriptive statistics for the reference group

\begin{tabular}{lll}
\hline & Group A & Group B \\
\hline Lactulose excreted in urine (\%) & $0 \cdot 261(0 \cdot 03)$ & $0.657(0.15)^{\star}$ \\
L-Rhamnose excreted in urine (\%) & $5 \cdot 611(0 \cdot 60)$ & $3.703(1.05)$ \\
Lactulose: L-Rhamnose ratio & $0.048(0.005)$ & $0 \cdot 159(0.03)^{\star \star}$ \\
Antigliadin antibodies above & 0 & \\
$\quad$ normal range (\%) & 0 & 100 \\
Altered breath hydrogen test (\%) & $39 \cdot 2$ & $21 \cdot 4$ \\
\hline
\end{tabular}

All results are expressed as mean $(\mathrm{SEM}){ }^{\star} \mathrm{p}<0.05 ;{ }^{\star \star} \mathrm{p}<0.01$; Mann-Whitney test. the intestinal permeability test compared with intestinal biopsy. Sustained increases of up to 20 ppm above the basal value recorded in the first 30 minutes were considered suggestive of intestinal overgrowth. Any peak $>20 \mathrm{ppm}$ above the basal value occurring outside the reference range (65120 minutes) was defined as indicative of altered oro-caecal transit times. Results of the intestinal permeability test were expressed as the percentage ratio of ingested lactulose recovered in the urine to the percentage of ingested L-rhamnose recovered in the urine.

\section{STATISTICAL TREATMENT}

Statistical analysis was carried out with a statistical package ${ }^{24}$ that included the MannWhitney test, one and two factor analysis of variance, a diagnostic performance study (sensitivity, specificity), and the Youden index, which is a calculation of the total diagnostic value of a probe. Its formula is expressed as: 1 -(sensitivity+specificity) and it ranges from 0 to 1 .

Receiver operating characteristic (ROC) curves and the areas below the curves were also calculated. This procedure is based on the calculation of the area below the curve formed by the graphical representation of sensitivity (ordinate) and 1-specifity (abscissa). It represents the discriminating power of the diagnostic probe for every cut value of the test.

\section{Results}

\section{REFERENCE GROUP}

Table I shows descriptive statistics for the reference group. The difference between the intestinal permeability test values for subgroups A and B was statistically significant. One factor analysis of variance gave significant differences for the intestinal mucosal state and the test values $(\mathrm{F}=57.87, \mathrm{p}<0.05)$.

The figure shows the diagnostic performance characteristics of the permeability test related to the histological state of the mucosa in the diagnosis protocol for coeliac disease. It also shows the excellent discriminating power of the test, with a cut off for the maximum Youden index at 0.92 .

IgA and IgG antigliadin antibodies were outside the reference range in all patients in subgroup $B$ and below the upper limit of normality in all subjects in subgroup $A$.

When two factor analysis of variance was applied (intestinal permeability and breath hydrogen test), the results indicated that neither oro-caecal transit time nor the probability of

TABLE II Descriptive statistics for the adolescent group

\begin{tabular}{lll}
\hline & Group A & Group B \\
\hline Lactutose excreted in urine (\%) & $0.231(0.04)$ & $0.257(0.02)$ \\
L-Rhamnose excreted in urine (\%) & $5.899(0.59)$ & $7.268(0.49)$ \\
Lactulose:L-Rhamnose ratio & $0.039(0.008)$ & $0.041(0.005)$ \\
Antigliadin antibodies above & 0 & 0 \\
normal range (\%) & 0 & 20.5 \\
Altered breath hydrogen test (\%) & 10.25 & \\
\hline
\end{tabular}

All results are expressed as mean (SEM). 


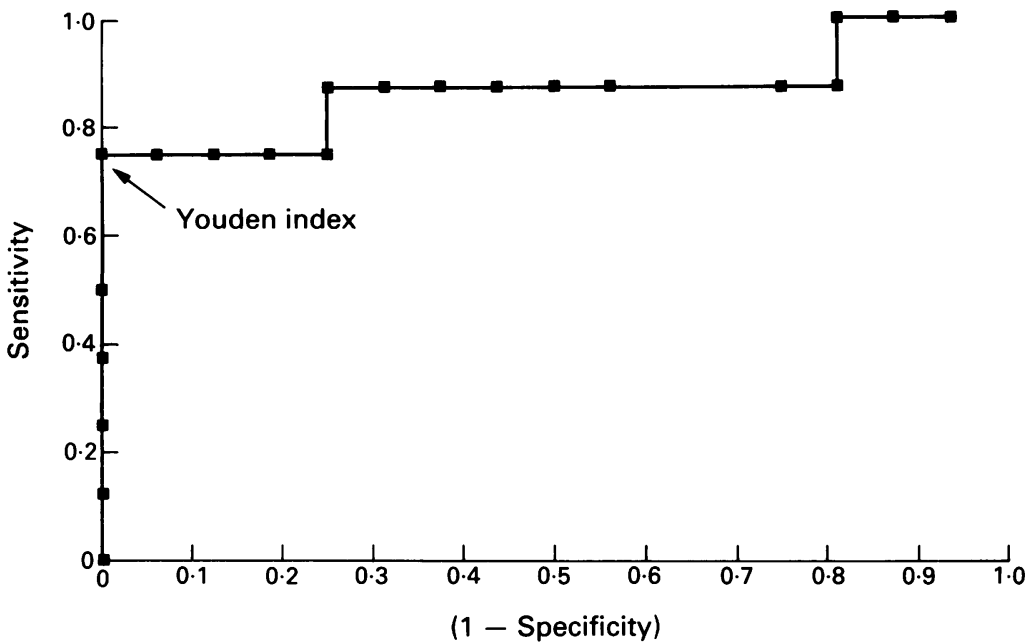

Figure: The area below the receiver operating characteristic curve delimited by symbols represents the good diagnostic performance of the intestinal permeability test, approaching 1 that is, the optimum value of the area. The arrow represents the cut off test value that renders the better diagnostic discrimination (maximum Youden Index) and is equal to a value of 0.092.

intestinal overgrowth influenced the intestina permeability results $(\mathrm{F}=1 \cdot 34)$. Severe histological damage was the only factor that affected intestinal permeability values. Results of the breath hydrogen test did not influence the relation between intestinal biopsy and intestinal permeability, and the variance for the two factor interaction was non-significant $(F=1 \cdot 0009)$.

\section{STUDY GROUP}

Table II shows results for the adolescents with coeliac disease. No differences in intestinal permeability values were established between subgroups I and II. None of the patients showed IgG or IgA antigliadin antibody concentrations above the upper limit for the reference range.

\section{Discussion}

Adequate therapeutic control is the main problem currently encountered in the treatment of coeliac disease. It is a well known fact that major dietary transgressions are normally accompanied by relapses, which are thus easy to recognise. Minor dietary regressions, which are difficult to detect, are factors that affect the future morbidity and mortality of patients with coeliac disease. Holmes et al studied development of coeliac disease in 210 patients over 11 years. ${ }^{2}$ They found that the patients who adhered strictly to a gluten free diet presented an excess morbidity similar to that for the general population $(1 \cdot 2)$; the excess mortality increased significantly in the patients who committed dietary transgressions, rising to 10.7 in the patients who followed a normal diet.

Adolescents with coeliac disease are able to evade parental dietary control and thus are more likely to commit dietary transgressions. ${ }^{4}$ As a standard for dietetic follow up testing, the ESPGAN has proposed the performance of antigliadin, ${ }^{25}$ antiendomysial, or antireticulin antibody determinations. Because of their low sensitivity, these tests are of questionable value in adolescents. ${ }^{10}$ "As a result in these patients verification of gluten induced villous atrophy can only be confirmed by means of intestinal biopsy. This involves subjecting patients to invasive techniques, with the consequent risk of rejection by patients.

It is therefore important to find a marker for slight dietary transgressions other than dietary questionnaires that is sensitive, non-invasive, and objective.

The intestinal permeability test is simple and is readily tolerated by patients. It has proved to be highly sensitive and specific in detecting severe villous atrophy. Our results for the reference group were in agreement with the results reported by other workers. ${ }^{26}$ They were normal in all the patients in subgroup $A$ (no histological damage) and abnormal in all the patients in subgroup B (severe atrophy). This excellent diagnostic performance supports the use of intestinal permeability as a screening test for secondary relapses due to severe dietary transgressions, as proposed by various researchers. ${ }^{27} 28$

Results on the sensitivity of intestinal permeability in cases of moderate histological changes in the intestinal mucosa have been contradictory, and its value in such cases remains unclear. ${ }^{1819}$ 29-31

All patients who presented evidence of severe dietary transgressions were excluded from this study. Our findings produced no differences in the results of intestinal permeability tests between patients who admitted having committed slight dietary transgressions and those who denied having committed any. Likewise, no differences were found in the behaviour of antigliadin antibodies in the two groups. Altered oro-caecal transit times or intestinal overgrowth did not decrease the diagnostic confidence of intestinal permeability tests.

In conclusion intestinal permeability tests can be useful in providing confirmation of diagnoses of coeliac disease and as a screening test for performing both the second and third confirmatory biopsies. Our results, however, confirm that intestinal permeability is not a valid marker for slight dietary transgressions. Three possible explanations for this are: (1) intestinal permeability is not sensitive enough to detect mild villous damage; (2) histological changes have not yet appeared in adolescents with coeliac disease; (3) after a period of meticulous compliance with a therapeutic diet, adolescents are able to tolerate variable amounts of gluten without exhibiting overt symptoms. ${ }^{32}$

More studies aimed at finding other markers of transgression are needed to enable clinicians to take steps to persuade patients to adhere to a gluten free diet and thus avert future malignant complications.

1 Polanco I. Enfermedad celiaca. In: Chantar C. Rhodes J, eds Actualidades en Gastroenterologia, vol 1. Barcelona: JR Prous, 1986: $151-4$.

2 Holmes GKT, Prior P, Lane MR, Pope D, Allan RN. Malignancy in coeliac disease-effect of a gluten free diet. Gu 1989; 30: 333-8.

3 Trier JS, Celiac sprue. $N$ Englf Med 1991; 325: 1709-19.

4 Shmerling DH, Franckx J. Childhood coeliac disease: a long term analysis of relapses in 91 patients. $\mathcal{f}$ Pediatri Gastroenterol Nutr 1986; 5: 565-9.

5 Kumar PS, Harris T, Walker-Smith J, et al. The teenage coeliac. Gut 1985; 26: A551.

6 Mulder CJJ, Tytgat GNJ. Coeliac disease and related disorders. Neth f Med 1987; 31: 286-99. 
7 Loga RFA, Rifkind EA, Turner ID. Mortality in coeliac disease. Gastroenterology 1989; 97: 265-71.

8 Diagnosis of coeliac disease [editorial]. Lancet 1991; 19: 92-3.

9 Mäki M, Holan K, Koskimies S, Hallstrom O, Visakorpi JK. Normal small bowel biopsy followed by coeliac disease. Arch Dis Child 1990; 65: 1137-41.

10 Savilahti E, Perkkio M, Kalimo K, Viander M, Varnio E, Reunala T. IgA antigliadin antibodies: a marker of mucosa damage in childhood coeliac disease. Lancet 1983; i: $320-2$.

11 Aurichio S, Greco L, Troncone R. Gluten-sensitive enteropathy in childhood. Pediatr Clin North Am 1988 35: 157-87.

12 Cobden I, Dickinson RJ, Rothwell J, Axon ATR. Intestinal permeability assessed by the excretion ratios of two molecules: results in coeliac disease. $B M \mathcal{F} 1978 \mathrm{i}: 1060$.

13 Strobel S, Brydon WG, Fergusson A. The cellobiose/mannitol test complements biopsy histopathology in clinical investigation of the jejunum. Gut 1984; 25: 1241-6.

14 Menzies IS, Laker MF, Pounder R, et al. Abnormal intestina permeability to sugars in villous atrophy. Lancet 1989; ii: $1107-9$.

15 Okabam SO, Clamp J, Cooper BT. Is small intestinal permeability increased or decreased in untreated coeliac disease? Clin Sci 1981;61: 39 .

16 Pearson ADJ, Eastham EJ, Laker MF, Craft JW, Nelson R. Intestinal permeability in children with Crohn's disease and Intestinal permeability in children with

17 Cobden I, Rothwell J, Axon ATR. Intestinal permeability and screening test for coeliac disease. Gut 1980;21: 512-8.

18 Hamilton I, Cobden I, Rothwell J, Axon ATR. Intestinal permeability in coeliac disease. The response to treatment and single-dose gluten challenge. Gut 1982; 23: 202-10.

19 Cooper BT, Ukabam SO. Changes in small intestinal permeability reflect the degree of mucosal abnormality in coeliac patients on a gluten free diet. Clin Sci 1982; 63: 21 .

20 Meewise G. European Society for Pediatric Gastroenterology and Nutrition. Diagnostic criteria in coeliac disease. Acta Pediatr Scand 1970; 49: 461-3.

21 Van de Kamer JH. Rapid method for the determination of fat feces. $\mathcal{F}$ Biol Chem 1949; 177: 347-55.
22 Lowry OH, Rosenbrough NJ, Lewiss FA, Randall RJ Protein measurement with the folin phenol reagent. $\mathcal{F}$ Biol Chem 1951; 193: 265-75.

23 Delhunty T, Hollander D. Liquid chromatographic method for estimating urinary sugars: applicability to studies of for estimating urinary sugars: applicability to stu
intestinal permeability. Clin Chem 1986; 32: 1542-4.

24 Fernandez-Peris E, Molinero-Casares LM, Morey Aboal E. Sigma. Base de datos bioestadistícos para un ordenador personal. Madrid: Horus Hardware, 1986.

25 Walker-Smith JA, Guandalini S, Schmitz J, Shermerling DH, Visakorpi JK. Report of the working group of the European Society of Paediatric Gastroenterology and Nuropean Revised criteria for diagnosis of coeliac disease. Arch Dis Child 1990; 65: 909-11.

26 Juby LD, Rothwell J, Axon ATR. Cellobiose/mannitol sugar est - a sensitive tubeless test for coeliac disease: results on 1010 unselected patients. Gut 1989; 30: 476-80.

27 Lifschitz CH, Shulman RJ. Intestinal permeability tests: are they clinically useful? F Pediatr Gastroenterol Nutr 1990; 10 . 283-7.

28 Juby LD, Rothwell J, Axon ATR. Lactulose/mannitol test: an ideal screen for celiac disease. Gastroenterology 1989; 96: 79 85.

29 Stenhammar L, Stomberg S. Intestinal permeability to Lactulose/L-rhamnose in children with celiac disease and other gastrointestinal disorders. I Pediatr Gastroenterol Nutr 1988; 7: 304-6.

30 Ford RPK, Menzies IS, Phillips AD, Walker-Smith JA Turner MW. Intestinal sugar permeability: relationship to diarrheal disease and small bowel morphology. $\mathcal{F}$ Pediat Gastroenterol Nutr 1985; 4: 468-74.

31 Nathavitharana KA, Lloyd DR, Brown GA, McNeish AS Urinary mannitol: lactulose excretion ratios and jejunal mucosal structure. Arch Dis Child 1988; 63: 1054-9.

32 Mäki M, Lähdeaho ML, Hällstrom $\mathrm{O}$, Viander M, Visakorpi JK. Postpubertal gluten challenge in coeliac disease. Arch Dis Child 1989; 64: 1604-7. 